\title{
Lógicas da JustificAÇÃo E QuASE-VERdAdE
}

\author{
Alexandre Costa-Leite
}

\begin{abstract}
Two kinds of justification logics are studied. Then, this article shows how the notion of quasi-truth can be defined in these systems.
\end{abstract}

Keywords: Justification; definitions of quasi-truth; Newton da Costa; Sergei Artemov.

\section{Introdução}

O conceito de justificação tem lugar de relevância central em qualquer teoria, independente de sua natureza. Sejam nas ciências (formais ou empíricas) ou na filosofia, justificar uma proposição fornece critérios para dizer o quanto um determinado enunciado se conecta com a verdade, a qual é aqui vista de modo padrão e tradicional como correspondência ou adequação do enunciado com a realidade. Ainda, dada uma proposição verdadeira, ela pode ser qualificada, quanto a sua força metafísica, como uma verdade possível, contingente ou necessária. Se um enunciado está justificado como sendo verdadeiro, ou se possui uma ampla variedade de justificações, então alguém tem boas razões para aceitá-lo. Grande parte das proposições que acreditamos não estão, contudo, justificadas plenamente, ou não possuem uma justificação total capaz de gerar certezas.

Há muitos modos de se justificar uma proposição de forma a fazer com que alguém tenha elementos suficientes para aceitá-la, mesmo que provisoriamente, como verdadeira. Essas maneiras são métodos de justificação, alguns mais robustos que outros no sentido de que dão mais evidência acerca da verdade. Aqui uma lista incompleta: a demonstração de um teorema, o depoimento de uma testemunha em um processo, um exame de DNA ou de sangue, uma perícia, uma prova documental, uma verificação, uma experiência em laboratório, um objeto qualquer, ou até mesmo outra proposição.

As justificações podem ser divididas em duas categorias fundamentais: as matemáticas e as não-matemáticas. Cada uma delas determina o nível da justificação, isto é, o quanto esta faz uma dada proposição ser verdadeira. Com isso, em geral, existem as justificações fortes, completas e totais, embora também existam as justificações fracas, incompletas e parciais. A justificação total garante que a proposição justificada é uma verdade necessária ao passo que a justificação fraca garante apenas que a proposição seja uma verdade contingente. Uma demonstração de um teorema, por exemplo, é uma justificação mais forte que o depoimento de uma testemunha em

Principia 18(2): 175-186 (2014).

Published by NEL — Epistemology and Logic Research Group, Federal University of Santa Catarina (UFSC), Brazil. 
um processo jurídico. Isso acontece porque a demonstração dá ao teorema o caráter de verdade necessária, ao passo que o depoimento da testemunha apenas fornece alguns indícios para que um juiz ou um júri monte sua convicção, o depoimento é uma justificação bastante fraca, e com isso gera uma verdade apenas possível e nãonecessária. Assim, níveis de justificação implicam modos de verdade fundados nos conceitos metafísicos de necessidade, possibilidade e contingência.

Por um lado, parece natural dizer que se uma proposição foi demonstrada, então a certeza acerca da verdade dessa proposição é total. Isso significa que, uma vez verdadeira, a proposição não pode mais ser falsa, uma vez que foi demonstrada como verdadeira. Nesse sentido, a proposição não é simplesmente verdadeira, mas necessariamente verdadeira, ou seja, uma verdade necessária. Por outro lado, justificações não-matemáticas não são capazes de lidar com verdades necessárias, mas sim, somente, com proposições contingentemente verdadeiras. Se não existe uma demonstração acerca da verdade de uma proposição, então não há certeza. Logo, a proposição não merece ser reconhecida como necessariamente verdadeira.

A noção de justificação recebe uma abordagem formal, trata-se de um conceito que pode ser matematizado e qualificado em uma lógica. Pode-se fazer isso de dois modos distintos embora compatíveis. Neste artigo, esses dois tipos de lógicas são estudados para compreender a estrutura formal da justificação, bem como utilizados para redefinir conceitos divergentes de quase-verdade.

\section{Lógicas da justificação}

Há, basicamente, dois formalismos diferentes para representar sistemas lógicos para a justificação. O primeiro deles foi desenvolvido por Newton da Costa em (1999). Ele elaborou lógicas para modelar tanto a justificação fraca quanto a forte por via de operadores modais com um comportamento parecido com a necessidade das lógicas modais normais aléticas. O segundo tipo de lógicas da justificação foi desenvolvido por Sergei Artemov em (2008). Ele introduziu outra família de lógicas da justificação para aumentar o poder expressivo de lógicas modais epistêmicas, tratando a noção de justificação não como um operador modal, mas como um termo associado aos operadores modais, sobretudo aos conectivos de conhecimento.

\subsection{As lógicas da justificação de Newton da Costa}

Aquilo que foi apresentado por N. da Costa sobre as lógicas da justificação encontrase no livro (1999), e a investigação desta seção é baseada naquelas informações (p.31, 42, 43, 60-2). O objetivo do autor consiste em modelar o conhecimento científico:

Principia 18(2): 175-186 (2014). 
O conhecimento forte se dá no campo da lógica e da matemática, ao passo que várias formas do fraco aparecem no domínio da experiência. Por conseguinte, o conhecimento científico pode ser fraco ou forte, o segundo atingindo o seu mais alto grau de certeza nas ciências formais. (Nas reais, quase nunca há a mínima chance de se adquirir conhecimento vasto e absolutamente seguro. A certeza de um conhecimento se mede pelo grau de justificação que dele se tem. Daí se dizer que a lógica e a matemática possuem uma intensidade de certeza praticamente nunca atingida nas demais ciências). (da Costa 1999, p.43)

O que vai medir a qualidade do conhecimento, se ele é forte ou fraco, é então o "grau de justificação". Assim, N. da Costa infere que uma lógica da justificação forte serve para as ciências formais e uma lógica da justificação fraca serve para as ciências, em geral. Com o objetivo de apresentar essas duas lógicas, considere uma linguagem com um conjunto $\{\varphi, \psi, \ldots\}$ de esquemas de fórmulas, e operadores lógicos: $\neg$ (negação) e $\rightarrow$ (implicação). Dois operadores modais são adicionados à essa linguagem: $J$ e $J^{\prime}$ para representar a ideia de justificação forte e justificação fraca, respectivamente. A leitura de $J_{i} \varphi$ é alguém está fortemente justificado a crer em $\varphi$ e a leitura de $J_{i}^{\prime} \varphi$ é alguém está fracamente justificado a crer em $\varphi$. O índice $i$ - que representa o número de agentes em questão - será omitido, dado que todas as lógicas consideradas aqui são para um único agente. Os sistemas obtidos são lógicas modais semelhantes aos sistemas modais desenvolvidos na literatura e têm uma apresentação feita à la Hilbert.

A lógica para a justificação forte é chamada de $\boldsymbol{J}$ e é elaborada a partir da lógica proposicional clássica, com a adição dos seguintes esquemas de axiomas:

1a. $J(\varphi \rightarrow \psi) \rightarrow(J \varphi \rightarrow J \psi)$;

2a. $J \varphi \rightarrow \varphi$;

(RIJ) Se $\vdash \varphi$, então $\vdash J \varphi$.

O sistema acima difere em relação ao original proposto por N. da Costa para a justificação forte: ao invés do axioma 2a, o autor usa instrospecção positiva para a justificação forte: $J \varphi \rightarrow J J \varphi$. A opção aqui pelo axioma 2 é que ele revela o fato essencial para a justificação forte de que se existe uma justificação total de $\varphi$, ou seja, uma demonstração, então $\varphi$ é verdadeira. Ainda, a lei (3a) $J \varphi \rightarrow \neg J \neg \varphi$ faz parte da axiomática original, isto é, não é possível justificar uma fórmula e sua negação (ver o argumento em da Costa (1999), p.61). Contudo, (3a) pode ser obtida a partir de (2a). Então, a lógica para a justificação forte é simplesmente a lógica modal normal KT.

Uma lógica da justificação fraca deve (i) representar justificações não-matemáticas, isto é, justificações incompletas e parciais que não garantem que o enunciado seja uma proposição necessariamente verdadeira. Por isso, para a justificação fraca 
não devem valer nem o axioma (2a) nem sua consequência (3a). Além disso, (ii) deve ser razoável para a justificação fraca que duas proposições contraditórias sejam justificadas. Há, de fato, algumas maneiras de fazer com que a lógica tenha essas propriedades desejáveis (i) e (ii). Uma maneira imediata e mais evidente é substituir a base clássica da lógica da justificação forte por algum sistema paraconsistente, deletando ainda a lei (2a). Outro modo é tratar a justificação fraca não como um operador tipo $\square$, mas antes tipo $\diamond$ ou até mesmo como contingência. N. da Costa não optou por nenhuma das soluções acima, mas sim pela escolha de um sistema modal com base clássica, embora mais fraco que a lógica $\boldsymbol{J}$. Deste modo, a lógica para a justificação fraca $J^{\prime}$ é dada por (ver da Costa 1999, p.42 e p.62):

1b. $J^{\prime}(\varphi \wedge \psi) \rightarrow\left(J^{\prime} \varphi \wedge J^{\prime} \psi\right)$;

2b. $J^{\prime} \varphi \longleftrightarrow J^{\prime} J^{\prime} \varphi$;

$\left(\mathrm{RIJ}^{\prime}-1\right) \quad$ Se $\vdash \varphi \leftrightarrow \psi$, então $\vdash J^{\prime} \varphi \leftrightarrow J^{\prime} \psi$;

$\left(\mathrm{RIJ}^{\prime}-2\right)$ Se $\vdash \varphi$, então $\vdash J^{\prime} \varphi$.

N. da Costa adiciona ainda uma cláusula que indica que a lógica deve aceitar um conjunto específico de fórmulas $J^{\prime} \varphi$ (sendo $\varphi$ uma sentença considerada como verdadeira em uma área de investigação, ou seja, uma teoria).

Dadas as lógicas $\boldsymbol{J}$ e $\boldsymbol{J}^{\prime}$, é possível ainda relacionar os dois sistemas por via da fusão de lógicas modais, isto é, por via de um método para combinar lógicas muito simples que consiste basicamente em gerar um novo sistema contendo todos os axiomas e regras de inferência das duas lógicas. O acréscimo de um axioma de interação para modelar relações entre a justificação forte e fraca é sugerido (ver da Costa 1999, p.42):

(INT) $J \varphi \rightarrow J^{\prime} \varphi$.

Tanto a lógica $\boldsymbol{J}$ quanto a lógica $\boldsymbol{J}^{\prime}$ possuem semânticas de Kripke, com a diferença de que o sistema $\boldsymbol{J}$ precisa da reflexividade para as relações de acessibilidade. Dado um modelo de Kripke definido de modo usual como $\langle W, R, v\rangle$, onde $W$ é um conjunto não-vazio de mundos possíveis, $R \subseteq W \times W$ é uma relação de acessibilidade, e $v: F \rightarrow \rho(W)$ é uma valoração que vai do conjunto $F$ de fórmulas para partes dos mundos, a condição de verdade para a justificação é: $w \vDash J \varphi$ se, e somente se, para todo $w^{\prime}$ tal que $w R w^{\prime}, w^{\prime} \vDash \varphi$. Se $R$ é reflexiva, tem-se a justificação forte, pois o axioma $J \varphi \rightarrow \varphi$ é satisfeito. Se $R$ não é reflexiva, tem-se a justificação fraca.

Como dito, a diferença crucial entre os dois tipos de justificação reside no fato de que a justificação forte não permite que uma proposição $\varphi$ seja justificada e sua negação $\neg \varphi$ também seja justificada, ao passo que a justificação fraca aceita esse tipo de situação. Bastante plausível, dado que se existe uma prova de um teorema $A$, e se a prova está correta, então $A$ tem uma justificação plena e não pode valer a 
negação de $A$, isto é, não pode existir uma justificação para a negação de $A$. Isso para as justificações fortes. Quando a justificação é fraca, faltam indícios totais para afirmar a verdade de uma dada proposição, então é perfeitamente aceitável que exista uma justificação para uma proposição e uma justificação para a negação dessa proposição. Isso acontece, frequentemente, com sentenças acerca da realidade. Em um processo jurídico, por exemplo, existem algumas vezes justificações para proposições contraditórias.

A partir da constatação de que existem então dois tipos de justificação e, com isso, dois tipos de conhecimento, as lógicas da justificação são combinadas com outros formalismos modais, tanto com uma lógica da verdade, quanto uma lógica da crença para, enfim, capturar definições desses dois tipos conhecimento (detalhes estão no livro da Costa (1999)). O trabalho de Newton da Costa sobre as lógicas da justificação é pioneiro e permite uma abordagem formal de vários problemas e paradoxos epistêmicos.

\subsection{As lógicas da justificação de Sergei Artemov}

Uma outra hierarquia de lógicas da justificação foi apresentada por Sergei Artemov. A presente seção que é descritiva, e contém uma simplificação na exposição, é elaborada com base no artigo Artemov (2008), o qual contém um relato acerca do surgimento e dos diferentes estilos de lógicas da justificação. Artemov inicialmente se concentrou em desenvolver uma lógica das provas, e chamou esse sistema de $\boldsymbol{L P}$ - logic of proofs. Todavia, essa lógica captura somente a justificação forte, ou seja, a ideia de demonstração matemática, apodítica, e se mostra insuficiente para a modelagem da justificação fraca. Então, Artemov desenvolveu famílias de lógicas da justificação incorporando tanto a justificação forte, total, quanto a justificação fraca, parcial. A sua antiga lógica das provas acaba sendo um sistema dessa família de lógicas da justificação (ver Artemov (2008), p.494).

Considere um conjunto de termos justificacionais $j, j^{\prime}, j^{\prime \prime} \ldots$; o formalismo $j: \varphi$ significa que jé uma justificação para $\varphi$ ou ainda existe uma justificação $j$ de $\varphi$. Duas operações são definidas para termos justificacionais, quais sejam: aplicação (.) e monotonicidade $(+)$. Essas operações são regidas pelos axiomas seguintes (eles podem ser usados para modelar justificações quaisquer que implicam ou não verdade), ambos estendendo a lógica proposicional clássica. Deste modo, a lógica básica da justificação $J_{0}$ é dada por (ver Artemov 2008, p.483):

$$
\begin{aligned}
& \text { 1c. } j:(\varphi \rightarrow \psi) \rightarrow\left(j^{\prime}: \varphi \rightarrow\left(j . j^{\prime}\right): \psi\right) ; \\
& \text { 2c. } j: \varphi \rightarrow\left(j+j^{\prime}\right): \varphi \text { e ainda } j: \varphi \rightarrow\left(j^{\prime}+j\right): \varphi .
\end{aligned}
$$

Do mesmo modo que nas lógicas da justificação de N. da Costa, Artemov leva em consideração justificações fracas e fortes. Estas - mais uma vez — são as jus- 
tificações que são poderosas e que formalizam, de certo modo, a ideia de prova matemática ou lógica. Aquelas são justificações enfraquecidas, que não fornecem critérios definitivos para determinar a verdade das proposições. Uma propriedade essencial de uma justificação forte é que ela, sendo uma justificação de uma dada proposição $\varphi$, implica então que $\varphi$ é verdadeira. Artemov chama essa propriedade de factividade das justificações (ver Artemov (2008), pp.491-492). Essa qualidade aparece expressa na lei da factividade:

(LF) $j: \varphi \rightarrow \varphi$.

(LF) não vale no sistema básico de lógica da justificação e, daí, tal lógica é perfeita para pensar justificações fracas. Quando estamos pensando em demonstrações matemáticas, a lei da factividade da justificação deve ser usada porque ela captura a noção fundamental de que se algo foi demonstrado, então é verdadeiro. Nesse caso, se (LF) for adicionada à lógica básica da justificação, tem-se o sistema $\boldsymbol{J} \boldsymbol{T}_{\mathbf{0}}$.

A contraparte semântica para o sistemas de justificação de Artemov funciona da seguinte forma: para darmos condições de verdade para as fórmulas, é preciso fazer uso da definição de um modelo de Kripke — tal como anteriormente (p.178) — com um ingrediente extra capaz de compreender a ideia de evidência admissível (ver a construção detalhada em Artemov (2008), p.488-9). Dado um modelo de Kripke $\langle W, R, v\rangle$, um componente $E$ é adicionado: $\langle W, R, E, v\rangle$ tal que $E$ é uma "função de evidência admissível", isto é, dada uma justificação $j$ e uma fórmula $\varphi$, a função de evidência admissível apresenta o conjunto de mundos onde $j$ é, de fato, uma justificação para $\varphi: E(j, \varphi) \subseteq W$. Assim, a condição de verdade para uma fórmula $j: \varphi$ em um mundo $w$ é a seguinte: $w \vDash j: \varphi$ se, e somente se, $w \in E(j, \varphi)$ e, para todo $w^{\prime}$ tal que $w R w^{\prime}, w^{\prime} \vDash \varphi$.

O trabalho desenvolvido por Sergei Artemov é bastante inovador e possibilita uma análise totalmente inédita de várias questões em epistemologia.

\section{Definindo quase-verdade por via das lógicas da justificação}

As lógicas da justificação - nas duas abordagens — podem ser usadas para definir e pensar o conceito de quase-verdade. Este foi proposto por Newton da Costa em (1986) porque, em geral, quando estamos fora da matemática e da lógica, não podemos falar exatamente em verdade necessária, mas somente em verdade contingente, isto é, quase-verdade. A noção de verdade nas ciências, defende Newton da Costa, deve ser substituída pela noção de quase-verdade (ver da Costa (1999), (1986) e Mikenberg, da Costa e Chuaqui (1986)). Da Costa pensou a noção de quase-verdade usando a ideia de Tarski, mas nas estruturas para modelar a quase-verdade, nem todas as fórmulas da linguagem possuem condições de verdade precisamente de-

Principia 18(2): 175-186 (2014). 
finidas, uma vez que para alguns objetos do domínio que não é possível dizer se estão ou não em determinada relação. Com o objetivo de obter essa diminuição da força metafísica do conceito - a verdade deixa de ser necessária para ser verdade contingente - da Costa propôs as noções de estrutura parcial e relação parcial:

Com essa noção de relação parcial, representamos as informações de que dispomos acerca de certo domínio do conhecimento, e mapeamos as regiões que necessitam de investigação adicional ... Desse modo, é possível, em certa medida, acomodar formalmente a "incompletude" das informações existentes no domínio científico. (da Costa \& Bueno 2006, p.654)

Essa tecnologia permite modelar domínios de conhecimento que possuem fatos desconhecidos e que apresentem necessidade de aumento do grau de justificação disponível. Vários estudos têm sido feitos desde então sobre a quase-verdade (para uma abordagem acerca da filosofia envolvendo o conceito de quase-verdade, ver Krause (2009). Para as relações entre o conceito de quase-verdade com a paraconsistência, assim como uma generalização daquele, ver o trabalho Silvestrini (2011)). Otávio Bueno e Edélcio de Souza argumentam que:

A versão de da Costa da quase-verdade é apresentada como uma noção enfraquecida de verdade, apropriada para a "parcialidade" e a "abertura", tipicamente encontradas na ciência e na vida cotidiana. De fato, nós dificilmente (se alguma vez) temos à nossa disposição informação completa acerca de um domínio qualquer que estamos investigando. (Bueno \& Souza 1996, p.188, tradução deste autor)

Essa informação incompleta pode ser concebida como uma falha no processo de justificação da proposição. Daí, é mais conveniente pensar a quase-verdade como ligada ao conceito de contingência antes que ao conceito de possibilidade, e jamais como necessidade, pois existem proposições possíveis que são também necessárias, ao passo que uma proposição contingente nunca implica necessidade. Se uma fórmula $\varphi$ é contingente, então ela é possível e sua negação também o é. Reduzir quaseverdade à possibilidade deixa espaço para dizer que existem quase-verdades que são, talvez, verdades necessárias, mas essa ideia não caracteriza aquilo que inicialmente pretendemos com o quase. A menos que seja desejável a existência de quaseverdades que podem se transformar em verdades necessárias, o caminho deve ser o de dizer que uma quase-verdade é uma verdade contingente. As definições apresentadas abaixo de quase-verdade têm os ingredientes de parcialidade, abertura e informação incompleta. Quando existe sucesso na justificação, quando estamos perante uma demonstração, portanto, podemos falar em verdade (necessária). Quando há falha no processo justificativo, somos levados ao domínio da quase-verdade. Uma justificação forte gera verdades necessárias ao passo que justificação fraca garante apenas verdades contingentes.

Principia 18(2): 175-186 (2014). 
Se temos dois tipos de lógicas da justificação, é natural que tenhamos dois modos para definir quase-verdade usando essas lógicas. A lógica $\boldsymbol{J}^{\prime}$ da justificação fraca captura a noção de quase-verdade da maneira seguinte:

(QV1) Uma fórmula $\varphi$ é uma quase-verdade se, e somente se, $J^{\prime} \varphi$ e $J^{\prime} \neg \varphi$.

Aqui o fato de a justificação ser fraca permite que existam justificações para proposições quase-verdadeiras contraditórias (Isso corresponde à ideia desenvolvida em Bueno e Souza (1996) de que existem níveis de quase-verdade, p. 195), contendo, evidentemente, níveis de informação incompleta.

A quase-verdade pode também ser definida usando os termos justificacionais da lógica básica da justificação $J_{0}$ :

(QV2) Uma fórmula $\varphi$ é uma quase-verdade se, e somente se, $j: \varphi$ e $j$ é uma justificação parcial para $\varphi$.

Uma vez que não existe a exigência de que $R$ seja reflexiva, a justificação de $\varphi$ não implica a verdade de $\varphi$, isto é, não vale em $J_{0}$ a factividade das justificações. Esse fato faz com que essa lógica seja ideal para modelar o conceito de quase-verdade também do ponto de vista semântico. Se $\varphi$ é uma quase-verdade, então $j: \varphi$ não implica $\varphi$. Daí podemos dizer que se $\varphi$ é uma quase-verdade, então não possui uma justificação total, completa, e que implica verdade. Assim como a lógica da justificação fraca $J^{\prime}$, a lógica básica da justificação $J_{0}$ pode ser pensada como uma lógica da quase-verdade.

A quase-verdade relativa a um mundo $w$ pode ser definida usando a ideia de modelo de Kripke enriquecido para as lógicas da justificação de Artemov:

(QV3) Uma fórmula $\varphi$ é uma quase-verdade em $w$ se, e somente se, $w \vDash j: \varphi$, $w \in E(j, \varphi)$ e, para todo $w^{\prime}$ tal que $w R w^{\prime}, w^{\prime} \vDash \varphi$.

Novamente, como não existe a exigência de reflexividade da relação $R$, está garantida a não-factividade e, portanto, a quase-verdade. Tanto para (QV2) quanto para (QV3) não há necessidade de impor a restrição de que $E(j, \varphi) \neq W$, assumindo que o conjunto $E(j, \varphi)$ fornece apenas os mundos nos quais $j$ é, realmente, uma justificação para $\varphi$, mas não se sabe se essa justificação é forte ou fraca.

Pode-se, de outro modo, definir níveis de justificação sem ordenação quanto a sua intensidade, ou seja, sem apresentar uma maneira de ordenar os graus de justificação. Para tanto, considere um conjunto $T J$ de termos justificacionais associados às fórmulas, e o intervalo $[0 ; 1]$. O nível de justificação $f: T J \rightarrow[0 ; 1]$ é tal que:

$$
f(j: \varphi)=\left\{\begin{array}{l}
1, \text { se } j \text { é uma demonstração de } \varphi ; \\
y, \text { para } y \in[0 ; 1[\text {, se } j \text { não é uma demonstração de } \varphi .
\end{array}\right.
$$

Com isso, surge a seguinte definição:

Principia 18(2): 175-186 (2014). 
(QV4) Uma fórmula $\varphi$ é uma quase-verdade se, e somente se, $f(j: \varphi)=y$, para $y \in[0 ; 1[$.

Tanto as lógicas da justificação no sentido de N. da Costa quanto as lógicas da justificação no sentido de S. Artemov podem receber um tratamento em termos de níveis de justificação. Para desenvolver o esquema acima para as lógicas de N. da Costa, basta substituir o termo justificacional pelo operador de justificação. Usando essa ideia, é plenamente possível que $J^{\prime} \varphi$ e $J^{\prime} \neg \varphi$, pois nesse caso, não sendo $J^{\prime}$ uma demonstração, seu nível de justificação é diferente de 1 . Daí, $J^{\prime} \varphi$ não implica $\neg J^{\prime} \neg \varphi$, pois, digamos, uma justificação de uma proposição pode ter nível 0,5 e a justificação da negação da proposição também ter o mesmo nível. Esse tipo de situação é bastante usual em se tratando de justificações fracas. Essa visão acerca da quase-verdade corresponde ao fato de que quando novas informações são adicionadas existe uma aumento de conhecimento, isto é, com o acréscimo de mais justificativas somos levados para níveis de quase-verdade.

A inspiração para a formulação dos níveis de justificação são os graus de crença na verdade examinados e explorados por N. da Costa em (1999), p.176-93. Os níveis de justificação correspondem, aproximativamente, à ideia de probabilidade objetiva de uma dada proposição, mas esses conceitos não devem ser confundidos, dado que uma proposição pode ter probabilidade 1, digamos, um evento certo, apesar de ter nível de justificação 0 . O nível de justificação é algo epistêmico, diz respeito ao grau de informação que um agente tem para aceitar uma proposição, ao passo que probabilidade é algo ontológico, independente das informações que o agente têm acerca de uma dada proposição. Suponha, como um experimento de pensamento, que no futuro alguns teoremas que hoje em dia não existem serão demonstrados. Esses teoremas têm probabilidade 1, atualmente, mas nível de justificação 0 .

\section{Conectando lógicas da justificação}

Os dois tipos de lógicas da justificação estão relacionados. As lógicas $\boldsymbol{J}$ e $\boldsymbol{J}^{\prime}$ são sistemas modais que formalizam um agente tem uma justificação (forte ou fraca) para $\varphi$, ou ainda, o agente está justificado a acreditar em $\varphi$. Nessa segunda leitura, de fato a adotada em da Costa (1999), dois operadores modais estão envolvidos: justificação e crença. Tem-se, portanto, uma multimodalidade, um conceito híbrido. Contudo, a abordagem pode ser feita levando em consideração justificação como eminentemente monomodal. Isso não é problemático porque as lógicas da justificação de N. da Costa são, na realidade, estudadas junto com sistemas para a crença e para a verdade. Tais lógicas podem então receber um sistema de justificações com o uso de termos justificacionais. Essa relação pode ser explorada no interior da combinação de lógicas por via de axiomas de interação.

Principia 18(2): 175-186 (2014). 
Considerando uma linguagem que contenha simultaneamente tanto termos justificacionais quanto operadores de justificação, é possível, ainda, encontrar uma lei que faça a conexão - não esperada - entre os dois sistemas de lógicas da justificação. Observando as condições semânticas que definem $J \varphi$ e $j: \varphi$, assumindo uma relação de acessibilidade reflexiva para os dois operadores, nota-se que os termos de justificação à la Artemov são mais específicos que o operador de N. da Costa, pois para que exista uma justificação de uma fórmula é preciso que $w$ satisfaça a condição de pertencer às evidências admissíveis (e essa condição é o que faz toda a diferença em relação aos operadores). Apesar do fato de que o operador de justificação $J$ recebe uma abordagem ao estilo do quantificador universal e isso faz com que tal modalidade tenha uma leitura universal, Artemov exige que seu formalismo $j: \varphi$ tenha uma leitura existencial e seja visto como: existe uma justificação de $\varphi$ (ver Artemov (2008), p. 498). Isso nos leva a conjecturar uma lei de interação imediata:

$$
j: \varphi \rightarrow J \varphi
$$

Esse axioma contém uma relação que conecta os dois tipos de lógicas da justificação. Essa ligação sintática aparece na contraparte semântica com muita nitidez. Evidentemente, dado o axioma de interação (INT) e a transitividade, segue-se que:

$$
j: \varphi \rightarrow J^{\prime} \varphi
$$

Os dois axiomas interativos acima representam o fato de que se existe, de fato, uma justificação para uma determinada proposição, então um agente está justificado (forte ou fracamente) a acreditar nessa proposição. A conexão entre termos justificacionais e operadores de justificação é imediata e, apesar de as duas abordagens serem diferentes, elas estão intimamente relacionadas.

\section{Conclusão}

O tema da justificação ocupa lugar de destaque em qualquer área do saber. As proposições não podem ser meramente afirmadas, se se tem a pretensão de percorrer fielmente e com precisão os caminhos da investigação. Os enunciados devem ser justificados, porque somente assim é possível dizer o quanto eles se conectam com graus metafísicos da verdade. A definição de verdade para alguns intuicionistas liga enunciados (necessariamente) verdadeiros com a existência de uma demonstração (sobre as diferentes concepções intuicionistas acerca da verdade, ver Raatikainen 2004). Assim como a justificação forte garante a verdade (necessária), a justificação fraca garante a quase-verdade por via de um enfraquecimento no critério de verdade intuicionista que conecta prova e verdade: quase-verdades possuem justificações fracas, incompletas e parciais. Embora não existam demonstrações para a 
maior parte das proposições, e isto faz com que tais sentenças não sejam necessárias, mas somente contingentes e parciais, ainda assim pode-se falar, fora do âmbito das demonstrações, em quase-verdades.

N. da Costa propõe em (1999) uma nova definição de conhecimento, distinta daquela tradicional, embora similar: o conhecimento é visto como crença quaseverdadeira probabilisticamente justificada (definição apresentada na p.187). A ideia de conhecimento proposta, assim como definições acerca de probabilidade subjetiva e probabilidade pragmática não são examinadas aqui e, no presente momento, não é possível dizer em que medida a ideia de níveis de justificação corresponde ou não a essas outras noções de probabilidade desenvolvidas por da Costa. Ainda, há uma série de estudos que podem ser feitos na direção das conexões entre as duas abordagens acerca da justificação, como por exemplo, investigar lógicas combinadas contendo simultaneamente operadores de justificação munidos com termos justificacionais. Isso permite um acréscimo de poder expressivo ao operador $J$. Entretanto, esses e outros temas, pela complexidade envolvida, serão examinados somente em trabalhos futuros.

\section{Agradecimentos}

Este trabalho foi financiado pelo CNPq (Projeto Universal 486635/2013-9).

\section{Referências}

Artemov, S. 2008. The logic of justification. The Review of Symbolic Logic 1: 477-513.

Bueno, O; da Costa, N. 2006. Quase-verdade. In: João Branquinho, Desidério Murcho e Nelson Gomes (eds.) Enciclopédia de termos lógico-filosóficos. São Paulo: Martins Fontes, p.653-7.

Bueno, O; de Souza, E. 1996. The concept of quasi-truth. Logique et Analyse 153-154: 183199.

da Costa, N. 1986. Pragmatic Probability. Erkenntnis 25: 141-62.

- 1999. O conhecimento científico, 2a. edição. São Paulo: Discurso Editorial.

Krause, D. 2009. Newton da Costa e a filosofia da quase-verdade. Principia 13(2): 105-28.

Mikenberg, I; da Costa, N; Chuaqui, R. 1986. Pragmatic Truth and approximation to truth. The Journal of Symbolic Logic 51: 201-21.

Raatikainen, P. 2004. Conceptions of truth in intuitionism. History and Philosophy of Logic 25: 131-45.

Silvestrini, L.H. 2011. Uma nova abordagem para a noção de quase-verdade. Doutorado em Filosofia, UNICAMP. 
Universidade de Brasília costaleite@unb.br

Resumo. Dois tipos de lógicas da justificação são investigados. A partir desse estudo, este artigo mostra como a noção de quase-verdade pode ser definida nesses sistemas.

Palavras-chave: Justificação; definições de quase-verdade; Newton da Costa; Sergei Artemov.

Principia 18(2): 175-186 (2014). 